\section{Cultural psychiatry}

\section{4}

Two-eyed seeing as a philosophy to facilitate communication between indigenous counselors and psychiatry about mind and mental health

\author{
B. Mainguy ${ }^{1 \star}$ and L. Mehl-Madrona ${ }^{2}$ \\ ${ }^{1}$ Education Division, Coyote Institute - Canada, Ottawa, Canada and \\ ${ }^{2}$ Medical Arts And Humanities Program, University of Maine, Orono, \\ United States of America \\ ${ }^{\star}$ Corresponding author. \\ doi: $10.1192 /$ j.eurpsy.2021.303
}

Introduction: The term "two-eyed seeing" is spreading across North America as a concept for explanatory pluralism. The concept was brought into academic science by Albert Marshall, a M'iqmaq from Nova, Scotia, Canada. It speaks to the idea that indigenous knowledge is an equally valid way of conceptualizing a phenomenon as is contemporary science. Marshall's famous example compares a traditional M'iqmaq story about the origins of the large tides in the Bay of Fundy with contemporary oceanographic geology findings and simulations.

Objectives: We wanted to explore how this two-eyed seeing model could be applied to mental health to facilitate a dialogue between psychiatry and traditional cultural healers.

Methods: We reviewed the existing literature on two-eyed seeing within mental health care using PubMed, IndexMedicus, OneSearch, and Google Scholar. We presented a course on two-eyed seeing for indigenous mental health services and two-eyed seeing for addressing trauma in indigenous communities and surveyed the participants about the two-eyed seeing concept. We offered this course primarily to providers within indigenous communities and also for other interested counsellors.

Results: Participants in our trainings were enthusiastic about the role of two-eyed seeing for improving communication among indigenous providers and patients and non-indigenous providers. Most indigenous counselors had not heard of two-eyed seeing and were quite enthusiastic about its affirming nature and how it gave them a basis for dialogue with non-indigenous practitioners.

Conclusions: Two-eyed seeing allows a rich dialogue between European-derived practitioners and indigenous people that enabls each to appreciate the other's perspectives, leading to greater cooperation and collaborative treatment.

Disclosure: No significant relationships.

Keywords: Indigenous people; two-eyed seeing; explanatory pluralism; cross-cultural communication

\section{6}

Group psychological intervention for people affected by conflict in Central African republic

\author{
E. Dozio ${ }^{1 \star}$ and C. Bizouerne ${ }^{2}$ \\ ${ }^{1}$ Mental Health And Care Practices, Action Contre la Faim, Paris, \\ France and ${ }^{2}$ Mental Health And Care Practices, Gender And \\ Protection, Action contre la Faim, Paris, France \\ ${ }^{*}$ Corresponding author. \\ doi: $10.1192 /$ j.eurpsy.2021.304
}

Introduction: A large part of the Central African population has been exposed to potentially traumatic events as a result of the recent conflict, which has led to the breakdown of social ties.

Objectives: Faced with this situation, the NGO Action contre la Faim proposed a psychosocial intervention aimed at helping the displaced people to reduce their psychological suffering and strengthen individual and community resilience.

Methods: After psychoeducation sessions organized in communities affected by the conflict, people identified with traumatic symptoms are invited to participate in a psychological support intervention. The protocol used is based on the Problem Management Plus (PM+), developed by the WHO. The approach was adapted in groups to reach more suffering people and also to take advantage of the group dynamic in the possibility of recovering and developing better resilience.

Results: 946 IDPs in the country's capital, participated in the group intervention led by a team of paraprofessionals. Data collected from 111 participants show that after five weeks of intervention, there was a significant reduction in post-traumatic symptoms (PCL-5) and functional impairment (WHODAS). These results were confirmed during the post-intervention evaluation four weeks later. In addiction, participants declared that they had observed effects on their ability to live together in the community and to regain social cohesion.

Conclusions: This experience gives encouraging results with regard to the feasibility and replicability of the group protocol, taking into account specific cultural and contextual adaptations.

Disclosure: No significant relationships.

Keywords: PM+; Central African Republic; Cultural Psychology; social cohesion

\section{7}

\section{Cultural competence in modern global world:} Applications for mental health

\section{R. Shilko* and L. Shaigerova}

Faculty Of Psychology, Lomonosov Moscow State University, Moscow, Russian Federation

${ }^{\star}$ Corresponding author.

doi: 10.1192/j.eurpsy.2021.305

Introduction: Cultural competence and related terms began to appear in the 1960s in the context of the development of civil rights movements in many countries. The importance of research of cultural competence among mental health professionals is raised with the globalization trends of the modern world, when the growth of ethnocultural diversity, internal and external migration, temporary movement of people lead to intensification of intercultural interaction.

Objectives: The current study aims to reveal contemporary tendencies in cultural competence understanding and development.

Methods: Theoretical analysis and systematization of research publications in order to clarify concepts, models and applications of cultural competence.

Results: The following tendencies were revealed. Cultural competence continues to attract significant attention of researchers and practitioners, especially among the mental health specialists (psychologists, psychiatrists, psychotherapists) who work with representatives of different cultures. A number of similar concepts and their components have been proposed: cultural competence, intercultural communicative competence, cross-cultural competence, cultural intelligence, cultural awareness, cultural acceptance, intercultural sensitivity, intercultural adaptation, multicultural competence, multicultural orientation. The difficulties and limitations of 
existing models noted: a shift of attention to a specialist, but not to a client; borrowing static and absolutistic ideas about cultures, without consideration of cultures development and interaction.

Conclusions: There is a trend in contemporary global world for broad research and development of cultural competence that improve professional qualities of healthcare professionals and provide psychological assistance to representatives of different ethnic and culture groups, confessions and minorities. The reported study was funded by the Russian Foundation for Basic Research, project number 17-29-02506.

Disclosure: No significant relationships.

Keywords: mental health; cultural competence; global world

\section{Depressive disorders}

\section{8}

\section{Adolescent oral contraceptive use and future major depressive disorder}

\author{
A. De Wit ${ }^{1 \star}$, C. Anderl ${ }^{2}$, E. Giltay ${ }^{3}$, T. Oldehinkel ${ }^{1}$ and F. Chen ${ }^{2}$ \\ ${ }^{1}$ Psychiatry, University Medical Center Groningen/University of \\ Groningen, Groningen, Netherlands; ${ }^{2}$ Department Of Psychology, \\ University of British Columbia, Vancouver, Canada and ${ }^{3}$ Department \\ Of Psychiatry, Leiden University Medical Center, Leiden, Netherlands \\ ${ }^{*}$ Corresponding author. \\ doi: 10.1192/j.eurpsy.2021.306
}

Introduction: Previously reported associations between oral contraceptives (OCs) use and depression have been conflicting. Insight into the impact of analytical choices on the association may help to reconcile previous heterogeneous findings.

Objectives: We aimed to examine the association between adolescent OC use and subsequent depression risk in early adulthood analyzing all theoretically justifiable models.

Methods: Women from the prospective cohort study Tracking Adolescents' Individual Lives Survey (TRAILS) were included in this study. All justifiable associations between adolescent OC use (ages 16-19 years) and major depressive disorder (MDD) in early adulthood (ages 20-25 years) as assessed by the Diagnostic and Statistical Manual of Mental Disorders-IV oriented Lifetime Depression Assessment Self-Report and the Composite International Diagnostic Interview were tested.

Results: A total of 818 analytical models were analyzed in 534 adolescent OC users and 191 nonusers. Overall, there was a tentative association of adolescent OC use and an episode of MDD in early adulthood (median odds ratio $[\mathrm{OR}]_{\text {median }}=1.41 ; \mathrm{OR}_{\min }=1.08$; $\mathrm{OR}_{\max }=2.18$, permutation testing $\mathrm{p}$-value $1=.052$, and $\mathrm{p}$-value $2=$ .046 ), which was primarily driven by the group of young women with no history of $\mathrm{MDD}\left(\mathrm{OR}_{\text {median }}=1.72 ; \mathrm{OR}_{\min }=1.21 ; \mathrm{OR}_{\max }=2.18\right.$, both permutation testing $\mathrm{p}$-values $=.02$ ).

Conclusions: Adolescent OC use was associated with an increased risk for experiencing an episode of MDD, but only among women with no history of MDD in adolescence. Understanding the potential side effects of OCs will help women and their doctors make informed choices when deciding among possible methods of birth control.

Disclosure: No significant relationships.

Keywords: Oral contraceptives; adolescence; Depression

\section{9}

Exploring predictors of depressive symptoms in patients with multiple sclerosis: The effect of neuropathic pain, shame, and mindfulness

T. Carvalho ${ }^{1,2}$, L. Benedito $^{1}$ and C. Gomes ${ }^{3 *}$

${ }^{1}$ Psychology, Instituto Superior Miguel Torga, Coimbra, Portugal;

${ }^{2}$ Faculty Of Psychology And Educational Sciences, University Of Coimbra, Center for Research in Neuropsychology and CognitiveBehavioral Intervention (CINEICC), Coimbra, Portugal and ${ }^{3}$ Clínica de Saúde Psiquiátrica de Coimbra - Casa da Oliveira, Coimbra, Portugal

${ }^{*}$ Corresponding author.

doi: 10.1192/j.eurpsy.2021.307

Introduction: Multiple Sclerosis (MS) is a chronic inflammatory, immune-mediated, demyelinating disease of the central nervous system, with a progressive course. It is potentially disabling and affects mainly young adults. Depression is the mental disorder with the greatest comorbidity with MS and tends to worsen its symptomatology and course. However, knowledge about the predictors of depression in patients with MS is scarce.

Objectives: This preliminary study aimed to verify whether neuropathic pain (NP), internal (IS) and external (ES) shame and mindfulness predict depressive symptoms in patients with MS.

Methods: This cross-sectional study included a convenience sample of 95 patients diagnosed with MS and without other identified neurological diseases. Participants completed the Depression Subscale of the Depression, Anxiety and Stress Scales-21, the Analogue Pain Scale of the Pain Detect Questionnaire, the External and Internal Shame Scale, and the Mindfulness Subscale of the Self-Compassion Scale.

Results: All potential predictors exhibited significant correlations with depressive symptoms and significantly predicted this symptomatology in simple linear regression models. Thus, they were included as covariates in the multiple linear regression model. This model explained a high percentage of the variance of depressive symptoms (40.5\%) and identified NP, IS and mindfulness as significant predictors.

Conclusions: Interventions aimed at preventing/reducing depression in patients with MS should minimize IS and develop mindfulness and NP coping skills, in order to promote mental health in this target population and possibly prevent the exacerbation and progression of MS symptomatology.

Disclosure: No significant relationships.

Keywords: Multiple sclerosis; depressive symptoms; predictive model

\section{1}

Baseline EEG-correlates of responders/non-responders to combined antidepressive treatment including transcranial magnetic stimulation

\section{E. Iznak ${ }^{1 *}$, A. Iznak ${ }^{1}$ and I. Oleichik ${ }^{2}$}

${ }^{1}$ Laboratory Of Neurophysiology, Mental Health Research Centre, Moscow, Russian Federation and ${ }^{2}$ Clinical Department Of Endogenous Mental Disorders And Affective States, Mental Health Research Centre, Moscow, Russian Federation

${ }^{\star}$ Corresponding author. doi: 10.1192/j.eurpsy.2021.308 